\title{
Hacia una visión biopragmática de la conciencia humana*
}

\author{
Towards a biopragmatic view of human consciousness
}

\section{Para uma visão biopragmática da consciência humana}

\section{Angélica María Rodríguez Ortíz}

\section{Resumen}

Los diversos estudios que circundan en la literatura de la filosofía de la mente nos acercan a la comprensión de la conciencia desde estudios fisicalistas, naturalistas y eliminativistas, los cuales, en su mayoría, terminan por sucumbir en el problema del dualismo (herencia cartesiana). La teoría del emergentismo searleano realiza su aporte sobre la comprensión de la naturaleza de la conciencia desde la contribución

* El texto pertenece al proyecto de investigación sobre la incidencia de la conciencia en la elaboración de juicios morales, desde la filosofía de la mente en John Searle, en el cual se pretende identificar el papel del lenguaje y la conciencia en la elaboración de juicios morales y la constitución de la moral como una institución social. DOI:http://dx.doi.org/10.15332/s0120-8462.2017.0116.10

* Magister en Educación por la Universidad de Caldas (2008). Candidata a doctora en Filosofía por la Universidad Pontificia Bolivariana (Medellin, Colombia). Licenciada en Filosofia y Letras por la Universidad de Caldas (2007). Lider y docente del programa de maestria en Enseñanza de las Ciencias de la Universidad Autónoma de Manizales. Investigadora del grupo: SEAD-UAM en la línea de Actores y Contextos. Correo electrónico: angelica.rodriguez276@gmail.com 
de las neurociencias, sin caer en reduccionismos y presenta una alternativa de superación del dualismo. Pese a ello, al analizar la teoría naturalista de Searle se evidencia que esta resulta ser insuficiente para comprender la naturaleza del fenómeno mental, además de caer en un nuevo dualismo: naturaleza/sociedad. Razón por la cual, en este escrito se realizará un análisis de la teoría searleana de la conciencia, con el fin de proponer al lenguaje como elemento constitutivo de la naturaleza de este estado mental, y no como producto derivado de la mente. Es decir, se propone una concepción biopragmática de la conciencia; estudio en el que se sustenta que biología y pragmática del lenguaje son condiciones sine qua non para la existencia de niveles superiores de la conciencia y superar el dualismo natural/social.

Palabras clave: conciencia, lenguaje, mente, dualismo, biopragmática.

\section{Abstract}

The various studies surrounding the literature of philosophy of mind bring us closer to the understanding of consciousness from physicalists, naturalists and eliminativists studies, which, for the most part, succumb to the problem of dualism (Cartesian inheritance). The Searlean theory of emergentism makes its contribution on the understanding of the nature of consciousness from the contribution of neurosciences, without falling into reductionisms and presents an alternative to overcome dualism. Nevertheless, when analyzing Searle's naturalistic theory, it is evident that this is insufficient to understand the nature of the mental phenomenon, besides falling into a new dualism: nature/society. For this reason, this paper will analyze the Searlean theory of consciousness, in order to propose language as a constituent element of the nature of this mental state, and not as a product derived from the mind. That is, a biopragmatic conception of consciousness is proposed; study in which it is maintained that biology and pragmatics of language are sine qua non conditions for the existence of higher levels of consciousness and overcome the natural/social dualism.

Keywords: Conscience, language, mind, dualism, biopragmatic. 


\section{Resumo}

Os diversos estudos que existem na literatura da filosofia da mente nos aproximam à compreensão da consciência desde estudos fisicalistas, naturalistas e eliminativistas, os quais, na maioria, sucumbem no problema do dualismo (herança cartesiana). A teoria do emergentismo searleano realiza seu aporte sobre a compreensão da natureza da consciência com a contribuição das neurociências, sem reducionismos e apresenta uma alternativa de superação do dualismo. Apesar disso, ao analisar a teoria naturalista de Searle evidencia-se que esta resulta ser insuficiente para compreender a natureza do fenômeno mental, além de apresentar um novo dualismo: natureza/sociedade. Razão pela qual, neste texto se realizará uma análise da teoria searleana da consciência, com o objetivo de propor à linguagem como elemento constitutivo da natureza de este estado mental e não como produto derivado da mente. Aliás, propõe-se uma conceição biopragmática da consciência; estudo o qual afirma que biologia e pragmática da linguagem são condições sine qua non para a existência de níveis superiores da consciência e superar o dualismo natural/social.

Palavras-chave: consciência, linguagem, mente, dualismo, biopragmática. 


\section{Introducción}

Develar la naturaleza de la conciencia se ha convertido en un tema obligatorio para la filosofía de la mente. En palabras de Searle (1992), "una teoría de la mente implica una teoría de la conciencia"; y como lo expone Nagel (1974), "una teoría seria de la mente está obligada a realizar un estudio sobre la conciencia”. En este sentido, las teorías de la conciencia plantean estudios que permiten la comprensión del fenómeno; estudios que continúan enmarcados en el problema del dualismo heredado de Descartes, aun cuando la mayoría de los autores afirman en sus planteamientos la superación del mismo.

En los orígenes de la filosofía de la mente se puede reconocer que el dualismo de Descartes ha dado pie a diversas teorías en pos de comprender la mente humana y, dentro de ella, al fenómeno de la conciencia. La filosofía de la mente, enraizada en la fenomenología y en la filosofía analítica, ha postulado teorías desde lineamientos psicológicos y conceptuales en pos de develar la naturaleza de la conciencia y superar el dualismo heredado, de lo que Ryle (2000) denominó la doctrina oficial. Sin embargo, pese al gran esfuerzo realizado por pensadores como Sellars, Dennett, Churchland, Clarck, entre otros, el problema del dualismo continúa, y como lo expone Chalmers (1996) la naturaleza de la conciencia continúa siendo un misterio, por lo cual, el camino sigue abierto para la discusión.

Tal vez, Ryle (1955) y Bennett y Hacker (2008) han acertado en la búsqueda de la raíz del problema, al plantear que los problemas que sugiere el dualismo de la doctrina cartesiana se deben a errores conceptuales; puesto que en la literatura sobre la mente y sus procesos, es común encontrar que el lenguaje utilizado para describir los fenómenos mentales es usado en el mismo sentido al describir los procesos biológicos que dan origen a tales estados. Es decir, el mal uso del lenguaje desemboca en un nuevo problema: el reduccionismo y, en el peor de los casos, llega a eliminar todo fenómeno mental. En este sentido, se puede inferir que el lenguaje es un elemento fundamental, pues permite trazar los límites en la comprensión del fenómeno, evoca confusiones frente al conocimiento del mismo y será el que permita analizar los errores conceptuales, con el fin de superar el dualismo que el mal uso del mismo crea en los discursos existentes. Un ejemplo claro lo presentan Bennett y Hacker (2008) cuando exponen que: 
Entre los neurocientíficos de hoy es muy habitual hablar de que el cerebro percibe, piensa, adivina o cree, o de que uno de sus hemisferios sabe cosas que el otro ignora. La justificación suele ser que este tipo de discurso no es más que una mera forma de hablar. Pero esto es un grave error, porque la forma característica de la explicación en la neurociencia cognitiva contemporánea consiste en adscribir atributos psicológicos y el ejercicio (y las diferencias en el ejercicio) de capacidades cognitivas por parte de los seres humanos (p. 19).

Es decir que el uso inapropiado del lenguaje lleva a que se presenten falsas superaciones del dualismo de propiedades; ya que toman como lo mismo "cerebro" y "mente", aun cuando son dos conceptos diferentes. El problema se acrecienta cuando algunas de las teorías sobre la mente, con este uso del lenguaje, convocan al reduccionismo y al eliminativismo de la conciencia y en algunos casos de la mente. Con lo cual afirman superar el dualismo, ya que usan los conceptos indistintamente para atribuirle cualidades a la mente o al cerebro.

Pues bien, al revisar la literatura existente en el tema de la conciencia, se observa que la generalidad de los estudios, realizados por los teóricos de la mente, se enfocan en la superación del dualismo heredado; sin embargo, con ello no logran explicar la naturaleza de este estado mental. Rechazan el uso inadecuado de los conceptos y rechazan el dualismo de propiedades, para lo cual presentan como alternativa la reducción de la mente a la materia, pero no dan cuenta de la naturaleza de la conciencia y de la mente, pues para algunos de ellos tales estados ni siquiera existen. Es decir, sucumben en un problema mayor al negar la existencia de la conciencia como estado mental, pues afirman que el hombre es solo cuerpo. En las siguientes líneas se presentará de manera breve algunas concepciones sobre la conciencia, entre ellas la de Searle, quien se opone a tales concepciones; con el fin de comprender un poco los planteamientos de autores que superan el dualismo desde el reduccionismo o desde el eliminativismo, y acaban por no dar cuenta de la naturaleza del fenómeno. En un segundo momento se analizarán los doce rasgos de la naturaleza que propone este filósofo analítico y se develará la insuficiencia que presentan seis de estos rasgos al ser justificados desde la biología; en un tercer momento se presentará al lenguaje como elemento constitutivo de la naturaleza de la conciencia y complemento a la justificación que brinda la biología. Es decir, el lenguaje está en la naturaleza de tal estado mental. 


\section{El problema del reduccionismo}

Algunas teorías de la mente presentan soluciones al problema del dualismo sustentadas en el análisis del lenguaje. Las teorías plantean alternativas de abordar la mente y el estado de la conciencia desde el uso del lenguaje, reconociendo los errores conceptuales que abundan en el discurso. Sin embargo, para brindar tales soluciones al problema del dualismo heredado presentan alternativas reduccionistas. En esta corriente podemos encontrar autores como Sellars, Ryle, Dennett y Churchland, quienes sucumben en reduccionistas o bien conceptuales o bien materialistas.

\subsection{El análisis reduccionista conceptual de la conciencia}

Una de las corrientes conceptuales que se enmarca en la herencia de los estudios de Ryle es la que inicia con Wilfrid Sellars. La teoría sense datum esboza desde las inferencias semánticas la insuficiencia epistemológica de la teoría de lo dado. Su crítica a las percepciones como garantía del conocimiento le permite al autor abordar el tema del lenguaje, no solo desde la concepción epistemológica, sino en la comprensión de la conciencia y demás estados de la mente.

Ryle y Sellars inician una bifurcación en la comprensión de la conciencia, ya no como estado psicológico, sino como conducta verbal. Las manifestaciones verbales evidencian los estados mentales; ser consciente de... implica una conducta verbal que se manifiesta a través de proposiciones. No hay conciencia de $X$, sino se expresa el enunciado "soy consciente de X". Todo estado mental, incluidos la conciencia y las creencias, se presentan en forma de enunciados. Hablar de conciencia implica hablar de conducta verbal. Todo comportamiento mental es un comportamiento verbal.

Para Sellars (1956), el lenguaje es el único que permite hacer explícitos estados mentales como la conciencia y las creencias, entre otros. La conducta verbal es la única garantía de que existe algo que llamamos mente. Asimismo, el lenguaje es la garantía de nuestras creencias y la justificación de las mismas. El lenguaje permite hacer explícito el parecer, el creer o el ser consciente del sujeto. Su teoría sienta las bases, para lo que posteriormente Brandom expone en su teoría sobre dar y pedir razones. Así, pues, el concepto está presente no solo en el parecer, en el creer o en el ser consciente, sino en la explicación que se exige para sustentar el parecer, el creer o el ser consciente de algo. 
[...] Todo percatarnos de géneros, parecidos, hechos, etc., en resumen, de entidades abstractas (y, en realidad incluso, de entidades singulares), es un asunto lingüístico. De acuerdo con ella, en el proceso de adquisición del uso del lenguaje no se presupone ni siquiera el percatarse de los géneros, parecidos y hechos relativos a la llamada experiencia inmediata (Sellars, 1956, p. 63).

Las teorías del conceptualismo reducen los estados mentales a las conductas verbales y sustentan mente y conocimiento en el uso del lenguaje. Cada expresión, cada proposición o enunciado no es más que la manifestación de la conducta mental. No sería posible hablar de estados mentales si estos son pensados como un fenómeno psicológico, puesto que tal cosa no es más que la extensión del lenguaje mismo. Toda conciencia de entidades abstractas y de entidades particulares es una cuestión lingüística.

Las acciones mentales son la extensión de las categorías verbales que las expresan públicamente. El lenguaje hace posible y visible las acciones mentales. “[...] Los pensamientos que solo un usuario del lenguaje puede pensar son pensamientos para los que no puede concebirse ninguna expresión no lingüística [...]” (Kenny, 2000, pp. 176-177).

\subsection{El reduccionismo materialista}

En otra línea reduccionista se pueden encontrar el materialismo y, en su versión extrema, el eliminativismo. Ambas teorías acaban por suscribirse al reduccionismo materialista o fisicalismo. El cerebro y sus procesos acaban por ser la mente y sus estados. No hay diferencias entre uno y otro, puesto que lo observable es lo único que garantiza la existencia de algo. Mente y cerebro son lo mismo, por lo cual, los procesos neurobiológicos acaban por ser procesos mentales. En otras palabras, la mente queda reducida a la materia, y para el caso del eliminativismo extremo de Chruchland y Dennett, la mente no existe. La mente es un invento del lenguaje dualista. No existe más que el cerebro y sus procesos neuronales; eso es lo que llamamos mente.

La mayor complejidad para los fisicalistas al abordar estudios sobre la mente, radica en el misterio que convoca el problema heredado de Descartes mente-cuerpo. Es difícil entender que los pensamientos compartan la misma materia de la que está hecho el cerebro. “¿Cómo es posible que mis pensamientos y mis sentimientos quepan en el 
mismo mundo que las células nerviosas y las moléculas que componen mi cerebro?" (Dennett, 1995, p. 13). Un misterio, que según Dennett, lleva a que el internalismo sustentado por Nagel y Chalmers se convierta en una visión extrema del dualismo cartesiano. El misterio de la mente inaccesible debe ser superado y enfrentado desde los descubrimientos de la ciencia. Nada puede ser explicado en términos metafísicos, pues todas las explicaciones están dadas en términos de funcionamientos físicos del cerebro.

La comprensión del funcionamiento de los mecanismos biológicos de nuestro organismo da cuenta de la conciencia, ya no como un fenómeno o un estado mental, sino como una serie de procesos del cerebro. “[...] Los diversos fenómenos que conforman aquello que llamamos conciencia [...] son efectos físicos de las actividades del cerebro [...]" (Dennett, 1995, p. 62).

La solución fisicalista al problema del dualismo cartesiano consiste en aceptar los nuevos descubrimientos de la ciencia y los aportes de estas en torno al funcionamiento del cerebro. "Es difícil imaginar cómo puede ser que nuestra mente sea nuestro cerebro, pero no es imposible" (Dennett, 1995, p. 28). La explanación que brindan las leyes físicas sobre el funcionamiento del cerebro permite comprender que no hay tejidos milagrosos ni procesos de ficción e ilusión como se ha creído y se ha afirmado en el tema de la conciencia. Las experiencias conscientes no son más que procesos de percepción sensorial, pero el lenguaje genera un dualismo de propiedades que nos lleva a considerar que la mente es un ente diferente al cuerpo.

En términos dennettianos, la heterofenomenología exige del uso del lenguaje verbal para describir las experiencias conscientes desde unas bases científicas; ya que la información de las experiencias sensibles, perceptuales o producto de la reflexión no son producto de las experiencias conscientes per se, sino producto del cerebro; puesto que:

La tarea del cerebro es guiar al cuerpo que controla en un mundo de condiciones cambiantes y sorpresas repentinas, así que debe acumular información sobre este mundo y utilizarla con rapidez para "crear futuro", para producir anticipaciones a fin de andar un paso por delante del desastre (Dennet, 1984a; 1991b, p. 158) y esto es lo que se ha conocido como conciencia. 
No hay un único y definitivo "flujo de conciencia", porque no hay un cuartel general central ni un Teatro Cartesiano donde "todo se junta" para ser examinado por un Significador Central. En vez de este flujo (por amplio que sea), hay múltiples canales en los que circuitos especializados intentan, en pandemóniums paralelos, llevar a cabo sus propias tareas creando Versiones Múltiples a medida que avanzan. [...] La serialidad de esta máquina (su carácter "von Neumanniano") no es un rasgo de diseño preconfigurado, sino el resultado de una sucesión de coaliciones entre estos especialistas. [...] Parte de este diseño es innato y compartido con otros animales. Pero se ve ampliado, y a veces superado en importancia, por microhábitos de pensamiento que se han desarrollado en el individuo, en parte como resultado de la autoexploración individual y en parte como dones prediseñados de la cultura [...]. (Dennet, 1995, pp. 267-268).

La conciencia es explicada desde la evolución biológica en términos fundantes de la inteligencia artificial. En otras palabras, el fisicalismo de Dennett busca superar el dualismo cartesiano y derrocar la postura internalista de la mente y sus procesos, así como la teoría internalista del lenguaje, con el fin de proponer como sustento que todas las memas producto de la información y la cultura, y en parte producto del lenguaje, son las que fijan su residencia en el cerebro de cada individuo, conformando sus tendencias y con ello llegando a convertirlo en lo que hemos llamado: "Mente". Así, la mente no es más que una ilusión creada por el lenguaje y la tradición cultural. "El lenguaje infecta y modula nuestro pensamiento a todos los niveles. Las palabras de nuestro vocabulario son catalizadores que pueden hacer precipitar fijaciones de contenido mientras una parte del cerebro intenta comunicarse con otra" (Dennett, 1995, p. 314).

\subsection{El emergentismo biológico de Searle. El inicio de la superación del dualismo}

Las teorías reduccionistas y eliminativistas realizan un intento por superar el dualismo cartesiano; sin embargo, este acaba por ser insuficiente a la hora de comprender la naturaleza de la conciencia, puesto que sus justificaciones centradas o bien en el lenguaje o bien en el cerebro y el sistema nervioso manifiestan afán de dar solución al problema planteado por Descartes, lo cual conlleva a la eliminación de la conciencia como estado mental. En medio de la proliferación entre eliminativistas, internalistas, 
conceptualistas y materialistas hay una teoría que intenta además de superar el dualismo cartesiano, dar cuenta de la naturaleza de la conciencia, para lo cual, retoma los aportes de la neurofisiología, sin llegar al extremo reduccionista.

Para Searle (1992):

El cerebro causa ciertos fenómenos "mentales", tales como los estados mentales conscientes, y esos estados conscientes son, simplemente, rasgos del nivel superior del cerebro. La conciencia es una propiedad emergente, o de nivel superior, del cerebro en sentido lisa y llanamente inocuo de "nivel superior" y "emergente" en el que la solidez es una propiedad emergente de las moléculas $\mathrm{H}_{2} \mathrm{O}$ cuando están en una estructura de enrejado (hielo), y la liquidez, es de manera similar, a una propiedad emergente del nivel superior de las moléculas $\mathrm{H}_{2} \mathrm{O}$ cuando están, para decirlo de manera aproximada, rodando unas con otras (agua). La conciencia es una propiedad mental y, por tanto, física, del cerebro en el sentido en que la liquidez es una propiedad de sistemas de moléculas (p. 29).

La conciencia no es el cerebro mismo, pero sí un estado mental que emerge de los procesos biológicos que el cerebro realiza.

La concepción emergentista adoptada por Searle, permite explorar en la naturaleza de la mente, para dar cuenta de la naturaleza de los estados mentales en términos de la biología. La postura naturalista de Searle, hace posible la comprensión de la conciencia como un estado mental, que si bien emerge del cerebro no puede ser reducido a este. Searle, rescata el internalismo y la subjetividad de la conciencia y lo sustenta en el externalismo natural. Así, [...] la conciencia no es reductible del modo en que otros fenómenos son reductibles [...] su realidad es la apariencia; de modo que la finalidad de la reducción se perdería si intentáramos aislar la apariencia y definiéramos simplemente la conciencia en términos de la realidad física subyacente. Para Searle (1983), la conciencia consiste en las apariencias mismas (p. 131).

\section{Searle: doce rasgos de la conciencia}

En sus estudios sobre la conciencia, Searle (1983) expone algunos planteamientos iniciales, los cuales cambian sustancialmente en el estudio que el mismo autor publica en 1992. Los estudios sobre el redescubrimiento de la mente se enfocan en doce 
rasgos estructurales que componen la naturaleza de la conciencia; rasgos que se consideran fundamentales; los cuales son trabajados en un contexto enmarcados en la temporalidad y la sociedad. Nuestra conciencia se experimenta temporalmente, y otros agentes conscientes resultan especiales en la estructura de las experiencias conscientes que tenemos. Este reconocimiento de otros seres conscientes, con capacidades similares a las nuestras, es lo que lleva al autor a trabajar bajo la presuposición de un trasfondo. Entendido este como la capacidad que permite a un ser humano asignar un estatus especial a otros de su misma especie. El trasfondo se hace evidente en las formas de intencionalidad colectiva, en las acciones, en el seguimiento de normas culturales (Searle, 1990).

La presentación de estos doce rasgos aportan a la comprensión sobre la naturaleza de ese rasgo físico de nivel superior del cerebro que denominamos: conciencia.

1. Modalidades finitas: para Searle, la conciencia humana se manifiesta en un número de modalidades, las cuales se pueden clasificar en percepciones corporales y flujo del pensamiento. En las percepciones corporales se hallan todas aquellas percepciones físicas que llegan a través de los sentidos, así como las sensaciones físicas como el dolor y el cansancio, las experiencias visuales y todas aquellas que llegan por medio de los cinco sentidos que todos conocemos, así como las que se le atribuyen al sexto sentido, o lo que el autor denomina sense of balance (sentido del equilibrio). En el caso del flujo del pensamiento, el autor nos muestra que a este le atañe todo lo relacionado con las palabras e imágenes y otros pensamientos que se quedan por fuera de estas dos, como sentimientos, emociones, deseos e ideas para las cuales no bastan las palabras y las imágenes, para entender mejor esta parte, Searle plantea el ejemplo de lo que ocurre al pensamiento cuando decimos "se nos encendió la bombilla", es un flujo del pensamiento que se escapa a la explicación, tanto representacional, como proposicional.

2. Unidad: la unidad en las experiencias de la conciencia, es presentada desde dos dimensiones, la dimensión "horizontal” y la dimensión "vertical”. Para este autor, la unidad horizontal es la organización de las experiencias conscientes a través de tramos cortos de tiempo, la segunda tiene que ver con lo que el autor ha llamado memoria vertical, y es la que vincula los elementos que se dan al tiempo en un organismo consciente, por ejemplo, el hecho de darme cuenta de que tengo en frente un computador, estoy escribiendo y estoy sentada en una silla, son tres 
percepciones diferentes vinculadas en una columna unificada. La unidad, desde la horizontalidad y la verticalidad dan sentido a nuestras experiencias.

3. Intencionalidad: un rasgo fundamental de la conciencia es la intencionalidad, pues tal y como lo plantea Searle (1990), "la mayor parte de la conciencia, sino toda, es intencional". Es decir, que toda experiencia consciente lleva a que el estado del sujeto se dirija hacia alguna cosa, en este sentido, cuando un sujeto tiene un estado de conciencia, siempre tiene conciencia de algo, y ese "de" es la parte intencional. A lo anterior, se le suma que cuando se tiene un estado consciente, dice el autor, dichas experiencias siempre tienen una perspectiva, lo que implica ver un objeto "desde..." lo que lleva a ver en los estados conscientes como estados intencionales.

4. Sentimiento subjetivo: siguiendo el punto 3 , reconocer la intencionalidad de los estados conscientes nos lleva, según Searle, de manera natural al sentimiento subjetivo de nuestros estados conscientes. La subjetividad es un rasgo fundamental de la conciencia en esta teoría, es la que posibilita el fundamento filosófico de la misma.

5. La conexión entre conciencia e intencionalidad: en este punto para el autor, pese a lo expuesto en Searle (1983), existe una concepción conceptual entre intencionalidad y conciencia, por tal razón una teoría sobre intencionalidad exige una explicación de la conciencia.

6. La base figurativa, la estructura gestáltica de la conciencia: este rasgo nos permite reconocer, que similar a lo que ocurre con la teoría de la Gestalt, sucede con la conciencia, es decir, que cuando somos conscientes de... centramos nuestra atención en algo, pero ese algo está sobre un trasfondo que no es el foco de mi atención. En este sentido el trasfondo se concibe como las condiciones de límite de la conciencia, por las cuales, "el hecho de que nuestras percepciones normales estén siempre estructuradas está relacionado con la estructura de base figurativa de las experiencias conscientes" (Searle, 1992, p. 142).

7. El aspecto de la familiaridad: en este rasgo el autor nos remite a los rasgos 3 y 6 anteriormente mencionados, para proponer que en todo estado consciente hay cierta familiaridad, pues en el estado de conciencia intencional, es decir, conciencia "de...", hay unos presupuestos generales, dados por una posesión previa de un aparato suficiente para generar dicha conciencia y las estructuras de la 
organización que esta posee, lo cual me lleva a identificar la familiaridad de los objetos de los cuales soy consciente y sus relaciones conmigo. Para el autor, el hecho de ver mis zapatos, me llevan a, además de tener una experiencia visual de percepción, reconocerlos como míos. En este sentido la familiaridad no es una experiencia separada de la conciencia, sino que se da simultáneamente, aparece solo en el momento en que soy consciente. Para Searle el aspecto de la familiaridad tiene diversos grados. En un primer grado se encuentra la familiaridad que tiene el sujeto con los objetos, escenarios y gente con la que comparte a diario; en un segundo grado está la familiaridad en la que las escenas extrañas, los objetos, escenarios y gente son reconocidas fácilmente por el sujeto consciente. En este sentido el aspecto de la familiaridad es el que hace posible la organización $y$ orden de las experiencias conscientes, y las percepciones son tales solamente bajo el concepto de lo familiar.

Los siguientes rasgos están conectados entre sí: estructuración, percepción como el contorno del aspecto de toda la intencionalidad y el aspecto de la familiaridad. Las experiencias conscientes nos vienen estructuradas, y esas estructuras nos capacitan para percibir cosas bajo aspectos, pero esos aspectos están constreñidos por nuestro dominio de un conjunto de categorías, y esas categorías, que nos son familiares, nos capacitan en grados diversos para asimilar nuestras experiencias por nuevas que sean, a lo familiar (Searle, 1992, p. 145).

La familiaridad nos permite a su vez comprender, un poco, el hecho de poseer capacidades de trasfondo, capacidades que son realizadas de formas neurobiológicas y las cuales posibilitan que nuestras experiencias sean estructuradas.

8. Desbordamiento: este es el rasgo que permite reconocer que en los estados conscientes se refieren a algo que está más allá de su contenido inmediato. Es decir, el contenido inmediato tiende a rebosar el pensamiento, pues inmediatamente tiende a conectar unos pensamientos con otros; pensamientos que se relacionan directamente con el contenido, pero que no eran evidentes en el contenido mismo.

9. El centro y la periferia: este rasgo es el que permite distinguir entre las cosas que están en el centro de la atención, del sujeto consciente y las que están en 
la periferia ${ }^{1}$. Es decir, un sujeto puede estar consciente de varias experiencias, pero centra su atención en solo una. En este sentido, se podría hablar de cierta clasificación de los grados de conciencia, en la cual el foco de la atención requiere de un grado de conciencia más alto que el que concierne a otras experiencias simultáneas que tiene el sujeto.

10. Condiciones límite: este rasgo es el que permite al sujeto una localización espaciotemporal y sociobiológica de sus estados conscientes. Es decir, permite una localización, la cual no es el objeto mismo de la conciencia (centro) ni es la periferia.

11. Estados de ánimo: este rasgo es el que proporciona el tono o color que caracteriza la totalidad de un estado consciente o de una secuencia de estados conscientes, en este sentido no se trata de decir que los estados de ánimo son estados conscientes, sino que los estados conscientes son invadidos por los estados de ánimo y nos permiten ser conscientes "de..." con un tono que varía según el estado de ánimo.

12. La dimensión placer/displacer: este es el último rasgo que expone el autor, al hablar de la totalidad de un estado consciente. En este sentido se plantea que en la conciencia hay una dimensión de placer y displacer, pues independientemente del estado puedo cuestionarme si me gustó o no, es decir, supongamos que soy consciente del olor de un dulce, en este sentido puedo relacionar esta percepción consciente con la pregunta: $\underset{i}{ }$ Me gusta dicho olor? En este sentido el autor afirma que hay diferentes subdimensiones de la dimensión del placer, tales como: el entusiasmo, el aburrimiento, las náuseas, la irritación, la felicidad, entre otros. Algunos estudiosos de la mente han llegado a plantear que parte de estos rasgos han sido tomados de la obra de Husserl: Investigaciones lógicas I y II (1900- 1901), sin embargo, al hablar con el autor, en un encuentro en Berkeley (2015), Searle afirma no tener tal influencia, pues los rasgos que expone son producto de los estudios sobre neurociencias y su teoría de Actos de habla (1969) que lo incitaron a transitar por el misterio de la conciencia, y si bien -afirma el autor- "hay cercanía entre mi propuesta y la de Husserl, no es porque lo haya leído, sino por la universalidad de los mismos rasgos, que le son propios a la naturaleza del fenómeno de la conciencia, y al estudiarlo se hacen ostensibles".

1 En algunas ocasiones se ha llegado a confundir, por el uso del lenguaje cotidiano el centro y la periferia con conciencia e inconciencia. Por ello, es importante dejar en claro, que hablar de periferia no nos remite lógicamente a hablar de inconciencia. 
Se puede observar, que estos doce rasgos descritos por Searle nos llevan a comprender la conciencia como un fenómeno emergente de lo físico, un fenómeno de macronivel del cerebro, cuya realidad y apariencia depende de la subjetividad; de las percepciones y del flujo de pensamiento que tenga el sujeto consciente. Pese a ello, se mostrará que tal planteamiento resulta insuficiente, pues considerar la conciencia como un producto emergente de la biología no da cuenta de la naturaleza misma, ya que a dicho estado le es propio el lenguaje en su uso pragmático.

\section{Análisis de los doce rasgos searleanos de la conciencia}

Los estudios del emergentismo biológico de Searle, pese a ser los más representativos y presentar argumentos sólidos frente a algunos de sus opositores, resultan ser insuficientes a la hora de definir la conciencia tanto individual como colectiva en sus grados o niveles superiores, al brindar una explicación de la naturaleza de la conciencia desde la neurofisiología.

El apoyo científico que Searle encuentra en el estudio realizado por el neurocientífico Edelman (1989), le permite entender la conciencia como el resultado de los procesos biológicos. Vista así, la naturaleza de la conciencia es explicada en la relación entre los estímulos sensoriales, que se convierten en constituyentes paradigmáticos de la experiencia consciente, los cuales permiten diferentes experiencias perceptuales en distintas modalidades: vista, oído, tacto, olor, gusto, propiocepción (la percepción de nuestro propio cuerpo), quinestesia (la sensación de la posición del cuerpo) y las sinapsis en el córtex cerebral, las cuales permiten a través de una cadena de corriente neuronal, el flujo de pensamiento en alta o baja intensidad. Según Edelman, cada una de estas modalidades comprende muchas submodalidades distintas cuando buscamos específicamente un punto concreto en el flujo constante de entradas sensoriales al que estamos expuestos; por ello, la percepción se convierte en una actividad orientada a la acción y la conciencia aparece como producto del proceso cerebral. La conciencia entonces es contemplada en la experiencia pasiva del sujeto (Edelman, 1989). El estado de conciencia primaria, en la teoría inicial de Edelman, depende del funcionamiento cerebral y es considerado como un estado mental acabado, puesto que emerge del cerebro y permanece no como proceso. Sin embargo, los aportes de Edelman en 1989 han evolucionado, como él mismo lo expone en los estudios realizados en compañía de Tononi, expuestos en su artículo Consciousness and complexity (1998) y retomados, 
posteriormente, en su libro A universe of consciousness, how matter becomes imagination (2000). Los planteamientos iniciales, si bien son retomados, son complementados con nuevos aportes no solo de las neurociencias sino de la filosofía. En dichos estudios, estos científicos cambian un poco la concepción inicial de Edelman sobre la conciencia. Ya no se la ve como como el resultado del funcionamiento de cada cerebro individual, al menos no como un resultado acabado, sino como un proceso que emerge del funcionamiento de cada cerebro individual; en el cual, además de presentarse una conciencia como proceso de orden primario, también se reconoce la existencia de experiencias conscientes de orden superior.

Nuestra posición ha sido que la conciencia de orden superior, que incluye la capacidad de ser consciente en primer orden, depende de la emergencia de una capacidad semántica y, en último término, del lenguaje. De forma concomitante aparece un auténtico yo, nacido de las interacciones sociales, juntamente con conceptos del pasado y el futuro. Impulsados por la conciencia primaria y el presente recordado, podemos, por medio del intercambio de símbolos y de la conciencia de orden superior, crear narraciones, ficciones, historias (Edelman, 1989, p. 128).

Ahora bien, de los doce rasgos presentados por Searle en 1992 para definir la conciencia, es preciso decir que al menos seis de ellos no son producto de una emergencia meramente biológica, tal y como él lo sustenta. En la naturaleza de tales estados, el lenguaje es un elemento constitutivo. La pragmática del lenguaje da cuenta de la naturaleza del estado mental, no solo a través de las descripciones; sino que hace posible la existencia de los mismos. Pese a que Searle (2010), en Making the social world, afirma que "[...] los límites establecidos por un lenguaje son los límites establecidos por la mente" (p. 16). La experiencia muestra lo contrario: los límites del lenguaje determinan los límites de la conciencia.

Pues bien, el autor habla de modalidades infinitas: dentro de las cuales están los flujos de pensamiento. Flujos que son generados por el sistema neurofisiológico. Dentro de estas modalidades es válido admitir parte de lo expuesto por Searle; ya que es innegable que una parte de dicho proceso sea biológico; no obstante, tales pensamientos de flujo requieren de la experiencia directa de los sentidos, así como de niveles avanzados de pensamiento que exigen un buen uso del lenguaje y procesos racionales y reflexivos. Es decir, que tales flujos de pensamiento exigen de contenido, 
y este está dado por las experiencias y el lenguaje que las describe. No es posible aceptar un flujo de pensamiento si no hay un lenguaje que lo posibilite. El lenguaje en su uso pragmático es el que hace posible que existan las modalidades infinitas. Cuanto mayor sea el dominio en el uso del lenguaje, mayor es la posibilidad de que haya flujo de pensamientos.

Otro rasgo esencial de la conciencia, según Searle, es la unidad. El autor habla de unidad horizontal y vertical, así como de memoria a corto y largo plazo; elementos que no son posibles si no hay experiencia directa con el tiempo y el espacio. Dicha experiencia de tiempo y espacio exige a su vez, conceptos, no solo para nominar "tiempo" y "espacio", sino para realizar la relación unificada de las experiencias. El uso del lenguaje en las experiencias espaciotemporales son las que permiten las descripciones unificadas que logramos de las mismas. ¿Cómo se logra una unidad de las experiencias vividas si no se tienen un lenguaje que lo haga posible? Los recuerdos que tenemos en nuestra memoria son expresados y unificados gracias al lenguaje.

Un tercer rasgo, expuesto por este pensador, es la conexión entre conciencia e intencionalidad: la relación expuesta por Searle entre estos dos estados mentales es netamente biológica, pero en realidad está dada en el uso del lenguaje. Cualquier pensamiento consciente e intencional exige del uso del lenguaje. La conciencia en un nivel superior, que vaya más allá de la percepción básica, demanda el uso del lenguaje. Ser consciente de... exige al sujeto usar el lenguaje no solo para expresar el contenido de su experiencia consciente, sino para reconocer su estado como intencional. Para Searle, el lenguaje es derivado de la forma más básica de la intencionalidad humana. Sin embargo, hasta la forma más básica de intencionalidad humana está dada en términos de lenguaje; por lo cual, el lenguaje es propio a la naturaleza de la mente misma, está en su constitución. Es decir, en la naturaleza del pensamiento humano este está como una condición biosocial. No existe pensamiento sin lenguaje. Y este último se desarrolla en el uso en la pragmática, en la comunicación con el otro, en la vida social.

No existen estados intencionales sin lenguaje, el dirigirse intencionalmente hacia algo implica su uso, además de las razones, de por qué se dirige la atención a $\mathrm{X}$ y no a Z. Dirigir la atención a X y ser consciente de ello, demanda en el sujeto de niveles superiores de flujos de pensamiento, lo que a su vez exige del uso del lenguaje. Puesto que, a mayor dominio del lenguaje en su uso, mayor será el nivel de intencionalidad y de consciencia de un agente. Tal vez la conciencia perceptual, en su nivel más básico 
pueda ser explicada en términos emergentes, pero sin lenguaje no es posible que el sujeto sea realmente consciente de su entorno y de sí. Los rasgos más básicos de la conciencia requieren lenguajes básicos, bien sean bocetuales o verbales, y los niveles superiores de conciencia demandan del lenguaje en su uso, de una pragmática en la cual hay un seguimiento de reglas para que haya una comunicación coherente. Un uso correcto del lenguaje conlleva a mejores flujos de pensamiento.

Otro rasgo esencial de la teoría searleana es la base figurativa de la experiencia gestáltica. Esta base figurativa que Searle expone como algo biológico, requiere en mayor grado el uso del lenguaje, más que los demás rasgos mencionados. Puesto que lograr experiencias gestálticas exige al individuo procesos de racionalidad. No existe racionalidad si no hay un lenguaje que permita el desarrollo de dicha capacidad. Alcanzar la comprensión gestáltica de las experiencias implica un uso correcto del lenguaje que solo se logra en las experiencias socioculturales. Si no hay lenguaje, racionalidad y experiencia social, no hay cómo comparar la parte con el todo e integrarlas. Este rasgo en las neurociencias puede ser sustentado desde el proceso de abstracción, pero aun en los procesos iniciales de abstracción, para lograr representaciones mentales, el lenguaje tanto bocetual como proposicional es fundamental. Sin este no es posible tener experiencias gestálticas.

Familiaridad y condiciones de límite. Al igual que los rasgos anteriores, estos dos rasgos no pueden considerarse como algo meramente natural. ¿Cómo algo llega a ser familiar si no se ha tenido una experiencia de ello? Basta con revisar nuestras experiencias más básicas para darse cuenta de que los rasgos de familiaridad se dan en la medida en que se han tenido experiencias cercanas. Si la familiaridad fuera una cuestión biológica, no desconoceríamos tantos fenómenos. ¿Por qué unas cosas nos son familiares y otras no? Podría decirse que por las experiencias previas con el entorno social y cultural; experiencias en las cuales se requiere del lenguaje y la reflexión para el seguimiento de patrones sociales. En cuanto a las condiciones de límite, es preciso aclarar que el autor plantea dicho rasgo como el que hace posible la comprensión de los fenómenos sociobiológicos, y traza o demarca los límites entre el centro y la periferia. Cabría preguntarse, entonces, ¿cómo delimita la conciencia entre lo que es social y lo que no lo es si no hay experiencia y lenguaje? Este rasgo no puede ser producto meramente natural, su misma función implica el reconocimiento de lo social y con ello del uso del lenguaje. 
En otras palabras, de los doce rasgos expuestos por Searle, estos seis se quedan cortos al ser sustentados en términos meramente biológicos. Aceptar el emergentismo natural como una teoría satisfactoria que explica la naturaleza de la conciencia implicaría aceptar que como seres humanos tenemos un único estado de conciencia en un grado muy bajo, porque las neurociencias sustentan la conciencia como un estado generado en la corteza cerebral, estado que es estático, lo cual llevaría a aceptar que existe un solo nivel de experiencias conscientes; pero las vivencias propias como las compartidas a través del lenguaje nos muestra que las experiencias conscientes varían entre los sujetos al ser descritas, así como varían en sus grados. Hay experiencias conscientes de niveles superiores, como la toma de decisiones morales, sociales y políticas, por ejemplo; las cuales, frente a las simples experiencias de percepción básica, demandan en sujetos un mayor dominio del lenguaje en su uso pragmático. Tomar una decisión de manera consciente es una experiencia de nivel superior, a ser consciente de que en este momento tengo sed. Los niveles superiores de conciencia se hacen manifiestos en el uso del lenguaje, basta con observar a los bebés y a los niños cuando se inician en el uso del lenguaje para ver que sus experiencias conscientes van variando de nivel.

\section{Hacia una concepción biopragmática de la conciencia que supere el dualismo}

El lenguaje es parte constitutiva de la naturaleza de la mente. Su uso, en las experiencias de interacción social con y en el mundo, se convierte en un elemento fundante de tal estado; por lo cual, al momento de realizar un estudio formal de la conciencia, de la comprensión de los grados superiores de dicho estado mental y del desarrollo de la misma, se hace obligatorio volver sobre el lenguaje.

En tal sentido, se afirma que la naturaleza de la conciencia es biopragmática. Es decir, hay ciertos elementos que emergen desde la neurofisiología, pero otros que incluyen la interacción con el entorno social y cultural, una interacción con el mundo dada en el uso pragmático del lenguaje. El lenguaje es un elemento sin el cual no es posible hablar de conciencia, ni comprender su naturaleza. Por otra parte, aceptar la naturaleza biopragmática de la conciencia no implica decir que la conciencia se reduce al lenguaje, sino biología y lenguaje hacen posible la existencia de tal estado mental. Con ello no solo se supera el dualismo cartesiano, sino que, además, se supera el dualismo natural/social. 
El lenguaje, por ser parte constitutiva de la mente humana y por ser un elemento biosocial, permite la relación conciencia-mundo. La pragmática del lenguaje hace posible que la conciencia humana sea un estado en evolución y que se presenten diferentes grados en las experiencias conscientes. Ser consciente de una percepción de un objeto en frente de mí, difiere de la experiencia de ser consciente de algo rojo en frente de mí. Asimismo, la experiencia consciente del objeto rojo, que se presenta en frente de mí, difiere de cualquier experiencia consciente que tenga en el campo de la moral. El grado de consciencia aumenta en los ejemplos citados.

El lenguaje permite que la conciencia humana se presente en diferentes grados, porque constituye la naturaleza de la misma, además, influye en su desarrollo. Retomando a Sellars, es el elemento que hace posible la manifestación de la conducta mental a través de la conducta lingüística. El lenguaje dota de contenido la experiencia consciente del sujeto, no solo en la emisión de la misma, sino en su origen. El lenguaje nos permite estudiar la conciencia, abordarla no solo desde lo psicológico, sino desde lo conceptual y develar el papel del conocimiento en el desarrollo de la misma. De acuerdo con lo anterior, se puede inferir que mayores grados de conciencia exigen al sujeto mayor pragmática del lenguaje y mayor número de creencias. Los niveles superiores de conciencia demandan de un mayor y mejor uso del lenguaje. 


\section{Referencias}

Bennett, M., y Hacker, P. (2008). La naturaleza de la conciencia. Cerebro, mente y lenguaje. Barcelona, España: Paidós.

Clark, A. (2008). Supersizing the mind. Embodiment, action, and cognitive extension. Londres, Inglaterra: Oxford, University Press.

Chalmers, D. (1996). La mente consciente. En busca de una teoría fundamental. Barcelona, España: Gedisa.

Clarck, A., \& Chalmers, D. (1998). The extended mind. Analysis, 58, (pp. 7-19).

Churchland, P. (1981). Eliminative materialism and the propositional attitudes. Journal of Philosophy, 78, (pp. 67-90).

Churchland, P. (1984). Matter and conscienciouss: A contemporary introduction of the philosophy of mind. Barcelona, España: Gedisa.

Dennett, D. (1978). Brainstorms: Philosophical essays on mind and psychology. Cambridge, Inglaterra: MIT Press.

Dennet, D. (1995). La conciencia explicada. Una teoría interdisciplinara. Barcelona, España: Paidós.

Edelman, G. (1989). The remembered present: A biological theory of consciousness. New York: Basic Books.

Edelman, G., \& Tononi, G. (1998). Consciousness and complexity. Science, 282, (pp. 1546-1851).

Edelman, G., \& Tononi, G. (2000). A universe of consciousness: How matter becomes imagination. New York: Basics Books.

Kenny, A. (2000). La metafísica de la mente. Barcelona, España: Paidós.

Nagel, T. (1994). What is it like to be a bat? Philosophical rewiev, 83(4), 435-450.

Ryle, G. (2000). The concept of mind. Londres, Inglaterra: Penguin Group.

Searle, J. (1983). Intentionality. An essay in the philosophy of mind. Cambridge, Inglaterra: Cambridge University Press. 
Searle, J. (1992). The rediscovery of the mind. Cambridge, Inglaterra: MIT Press.

Searle, J. (1995). The construction of social reality. New York: The Free Press.

Searle, J. (1996). La naturaleza de la conciencia. Barcelona, España: Paidós.

Searle, J. (2010). Making the social world. The structure of human civilization. New York: Oxford University Press.

Sellars, W. (1997). Empiricism and the philosophy of mind. Cambridge, Massachusetts: Harvard University Press. 